\title{
Optimal control of entangling operations for trapped ion quantum computing
}

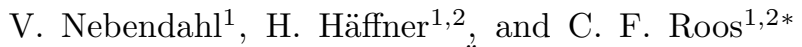 \\ ${ }^{1}$ Institut für Quantenoptik und Quanteninformation, Österreichische Akademie der Wissenschaften, \\ Otto-Hittmair-Platz 1, A-6020 Innsbruck, Austria and \\ ${ }^{2}$ Institut für Experimentalphysik, Universität Innsbruck, Technikerstr. 25, A-6020 Innsbruck, Austria
}

(Dated: October 25, 2018)

\begin{abstract}
Optimal control techniques are applied for the decomposition of unitary quantum operations into a sequence of single-qubit gates and entangling operations. To this end, we modify a gradient-ascent algorithm developed for systems of coupled nuclear spins in molecules to make it suitable for trapped ion quantum computing. We decompose unitary operations into entangling gates that are based on a nonlinear collective spin operator and complemented by global spin flip and local light shift gates. Among others, we provide explicit decompositions of controlled-NOT and Toffoli gates, and a simple quantum error correction protocol.
\end{abstract}

PACS numbers: 03.67-a, 32.80.Qk, 37.10.Ty

\section{INTRODUCTION}

Choosing the laws of quantum physics as the physical basis for constructing models of computation [1] allows for solving certain computational problems more efficiently as in models based on classical physics [2]. In the quantum circuit model, information is encoded in quantum bits (qubits) and manipulated by applying unitary operations acting on the joint state space of the qubits. It has been shown that arbitrary unitary operations can be broken down into sequences of elementary gate operations, consisting of single-qubit operations and entangling operations acting on pairs of qubits [3]. It is a non-trivial task to find optimum decompositions of unitary operations into a minimum number of elementary gates. Often, the controlled-NOT (CNOT) gate operation is chosen as the entangling operation. However, it has been shown that almost any entangling operation can be used for this purpose as well [4].

In experiments processing quantum information, the available physical processes determine the choice of the entangling gate. In the case of trapped ions manipulated by coherent laser light [5], a qubit is realized by encoding quantum information in a pair of long-lived internal states, consisting of hyperfine or Zeeman ground states, or in a combination of a ground state and a metastable state with an energy difference of a few electron volts. Gates acting on a single qubit are achieved by lasers coupling the qubit states by either dipole-forbidden singlephoton transitions or Raman transitions. Pairs of ions are entangled by qubit-qubit interactions mediated by a coupling to a vibrational mode of the ions' motion in the trap.

To achieve a gate on either a specific ion or a pair of ions within an ion string, two strategies are pursued:

1. The laser beam is tightly focussed so that it interacts only with a single ion at a time [6]. Then, a

*Electronic address: Christian.Roos@uibk.ac.at
CNOT gate or an equivalent entangling gate between an arbitrary pair of ions is achieved by a sequence of pulses with the laser addressed to either one or the other ion of the pair 7].

2. Alternatively, a laser with wider beam diameter is employed in combination with ions held in a segmented ion trap. In this approach, trap potentials are dynamically transformed to enable the transport of one or two ions into the interaction region with the laser beam. To entangle a pair of ions $i, j$, a bichromatic laser field is used to realize either a conditional phase gate [8] induced by an effective Hamiltonian $H_{P G} \propto \sigma_{z}^{(i)} \sigma_{z}^{(j)}$ or a Mølmer-Sørensen gate [9] induced by $H_{M S} \propto \sigma_{x}^{(i)} \sigma_{x}^{(j)}$ where $\sigma_{n}^{(k)}$ denotes a Pauli spin operator $\boldsymbol{\sigma} \cdot \mathbf{n}$ acting on the $k^{\prime}$ th ion.

Recently, the latter interaction has been used to entangle a pair of ions with fidelities of up to 99.3(1)\%, with a coupling mediated by the longitudinal center-of-mass mode of the ion string 10]. Employing the same kind of interaction to $N>2$ ions would realize a unitary operation $U_{M S}^{X}(\theta)=\exp \left(-i \frac{\theta}{4} S_{x}^{2}\right)$ with $S_{x}=\sum_{k=1}^{N} \sigma_{x}^{k}$, yielding an equal pairwise coupling between all ions in the string. In addition, using a single frequency resonant with the qubit transition instead of a bichromatic field coupling to the transitions' motional sidebands, the same laser beam could induce spin flips on all ions described by the unitary $U_{X}(\theta)=\exp \left(-i \frac{\theta}{2} S_{x}\right)$. This opens up the interesting prospect of performing arbitrary unitary operations by complementing these unitaries by single-qubit phase shift gates $U_{z}^{(k)}(\theta)=\exp \left(-i \frac{\theta}{2} \sigma_{z}^{(k)}\right)$ that could be induced by a tightly focussed off-resonant laser beam interacting only with the $k$ 'th ion. In this approach, no interferometric stability is required between the optical path lengths of the focussed and the wide beam. Moreover, the use of light shift gates facilitates addressing a single qubit in a string of ion qubits without introducing unwanted state transformations on the neighboring ions since the phase shift $\theta$ is proportional to the intensity of the laser field as 
compared to single-qubit spin flip gates where the rotation angle $\theta$ is proportional to the field amplitude [11]. In this way, unitary operations could be realized on a small group of ions without the need to split and rearrange the ion string in-between [12] and with more modest requirements regarding the spatial mode profile of the tightly focussed laser beam.

In this paper, we will use optimal control techniques to find decompositions of $N$-qubit gates into unitary operations induced by the set of Hamiltonians

$$
\mathcal{S}=\left\{S_{x}{ }^{2}, S_{x}, \sigma_{z}^{(1)}, \sigma_{z}^{(2)}, \ldots, \sigma_{z}^{(N)}\right\} .
$$

In contrast to similar applications of optimal control in nuclear magnetic resonance (NMR) experiments [13] and in laser-induced femto-chemistry [14, 15], we are interested in the case where only one of the Hamiltonians is applied at a time. As a consequence, the optimal control algorithm is required to find decompositions of a given quantum gate by sequences of laser pulses that interact either with all ions in the same way or with an individual ion.

The goal of this paper is to find gate decompositions of interest for current state-of-the-art ion trap experiments that are more efficient than decompositions based on gates acting on only one or two qubits at a time.

\section{BASIS SET OF OPERATIONS}

The mean-field interaction $S_{x}{ }^{2}$ acting on a string of $N$ ions entangles each ion qubit with each other ion qubit. Thus, on the one hand, application of this Hamiltonian endows us with the power to entangle arbitrary pairs of qubits. On the other hand, it induces entangling interactions that are not always desired. The situation we are encountering is somewhat similar to the one in NMR quantum computing where the system Hamiltonian $H_{\text {sys }}$ consisting of spin-spin interactions and chemical shifts leads to a system dynamics that needs to be controlled by radio-frequency fields interacting with single spins at a time. For this purpose, techniques have been developed for selectively switching off certain spin-spin interactions by refocussing techniques [16]. In the scenario we envision for the ion trap system, the role of the radiofrequency fields is taken over by laser pulses inducing single-qubit gate operations that are intermittently applied to particular ions.

Refocussing techniques are also applicable to the trapped ion system. For example, to entangle qubits 1 and 2 in a system of three qubits, the entangling pulse could be split into two parts and interleaved with a refocussing pulse to obtain the sequence $U=$ $U_{M S}^{X}(\pi / 4) U_{z}^{(3)}(\pi) U_{M S}^{X}(\pi / 4)$. The light shift pulse on the third qubit flips its phase and effectively reverses the entangling interactions with qubits 1 and 2. Substituting the last pulse by its inverse, results in a sequence $U=U_{M S}^{X}(-\pi / 4) U_{z}^{(3)}(\pi) U_{M S}^{X}(\pi / 4)$ that entangles qubits
1 and 2 with qubit 3 without inducing entangling interactions between 1 and 2 .

The set of Hamiltonians (11) is sufficient for generating arbitrary unitary operations on a string of $N$ qubits as can be shown by an explicit construction: A single-qubit x-rotation acting on qubit $k$ is generated by the spin echo sequence $U_{x}^{(k)}(\theta)=$ $U_{z}^{(k)}(-\pi) U_{X}(-\theta / 2) U_{z}^{(k)}(\pi) U_{X}(\theta / 2)$. Arbitrary singlequbit gates can then be performed when combining this operation with single-qubit phase shift gates $U_{z}^{(k)}(\theta)$. Similarly, an operation $U_{M S}^{X-x_{k}}$ that entangles all ions with each other except for qubit $k$ is produced from the $N$-qubit entangling gate $U_{M S}^{X}(\theta)$ by the pulse sequence $U_{M S}^{X-x_{k}}=U_{z}^{(k)}(-\pi) U_{M S}^{X}(\theta / 2) U_{z}^{(k)}(\pi) U_{M S}^{X}(\theta / 2)$. Substituting $U_{M S}^{X}$ in the above sequence by $U_{M S}^{X-x_{k}}$, a similar sequence is obtained that entangles all qubits except for two, and by induction, a two-qubit entangling gate is constructed between any pair of qubits $m$ and $n$ which together with arbitrary single qubit gates forms a universal set of gates.

While this construction shows that in principle arbitrary unitary operations are realizable by pulse sequences generated from the set $\mathcal{S}$, it is of no practical use. For the implementation of $N$-qubit gate operations, we are interested in finding pulse sequences that minimize gate errors occurring in ion trap quantum computing. Therefore, we will be searching for sequences having either a minimum number of (entangling) pulses or a minimum length in terms of the sum of pulse angles $\theta_{n}$ of the individual pulses.

\section{OPTIMAL CONTROL OF UNITARY TRANSFORMATIONS}

Optimal control techniques have been applied to the problem of generating specific unitary transformations 13, 14, 15, 17] with applications to systems as different as NMR, neutral atoms in optical lattices, Josephson junction qubits and trapped ions [13, 18, 19, 20]. To find decompositions of entangling ion trap gates in terms of pulses generated by Hamiltonians $H_{k}$ from $\mathcal{S}$, we modify a gradient-ascent algorithm that was developed by Khaneja et al. [13] in the context of NMR experiments. In their approach, a unitary transformation $U_{\text {target }}$ was searched for by constructing a unitary operation

$$
U=\prod_{m=1}^{M} U_{m}=\prod_{m=1}^{M} e^{\left(-\frac{i}{\hbar} \Delta t\left(H_{\mathrm{sys}}+\sum_{k=1}^{K} u_{k m} H_{k}\right)\right)},
$$

where $H_{k} \in \mathcal{S}$, that maximized the performance function $\Phi\left(\left\{u_{k m}\right\}\right)=\left|\operatorname{Tr}\left(U^{\dagger} U_{\text {target }}\right)\right|^{2}$. The authors noted that for small time increments $\Delta t$ the calculation of the gradient 21.

$$
\frac{\partial \Phi}{\partial u_{k m}} \approx-2 \operatorname{Re}\left(\operatorname{Tr}\left(\frac{i \Delta t}{\hbar} W_{m} H_{k} V_{m}\right) \operatorname{Tr}\left(W_{m} V_{m}\right)^{*}\right),
$$


with $W_{m}=U_{\text {target }}^{\dagger} U_{N} \cdots U_{m+1}$ and $V_{m}=U_{m} \cdots U_{1}$, could be efficiently carried out requiring only about $3 M$ matrix multiplications and about $K M$ calculation of traces. Then, a gradient-based algorithm was devised to increase the value of the performance function by modifying the control amplitudes

$$
u_{k m} \rightarrow u_{k m}+\epsilon \frac{\partial \Phi}{\partial u_{k m}}
$$

using a suitable step size $\epsilon$. Repeated application of the gradient calculation followed by updating the control amplitudes maximized the performance function and resulted in a unitary transformation realizing the target operation $U_{\text {target }}$.

There are a few important differences between coupled spin systems in NMR and in laser-manipulated strings of trapped ions. In the NMR context of ref. 13], the product of unitaries in (2) arises from a discretization of the time variable that required the approximation of a continuous control amplitude $u_{k}(t)$ by a stepwise continuous function with values $u_{k j}$. Restrictions to the values of $u_{k}(t)$ are only due to technical requirements like amplitude or bandwidth limitations of the radio-frequency equipment used for producing the control fields. In ion trap experiments, however, limitations exist for the values that the functions $u_{k}(t)$ can take on because the simultaneous application of different control Hamiltonians is either technically very challenging or physically impossible. In the former case, the simultaneous application of single qubit phase shift gates to more than a single ion would require control of the spatial profile of a laser beam inducing phase shifts by the ac-Stark effect. More importantly, in the latter case, the entangling interaction $S_{x}^{2}$ is produced by an effective Hamiltonian that precludes the simultaneous application of single-qubit phase shifts. As a consequence, the control functions need to satisfy the condition $u_{k}(t) u_{l}(t)=0$ for all $k, l$ at all times $t$. Therefore, the unitary transformation is naturally decomposed into a product of unitaries and Eq. (2) is replaced by

$$
U=\prod_{m=1}^{M} \exp \left(-i \theta_{m} H_{k_{m}}\right),
$$

where $\theta_{m}=\Delta t / \hbar u_{m}$ and $k_{m}$ labels the Hamiltonian from set $\mathcal{S}$ that is to be used for the mth pulse. As the system is stationary in the absence of laser interactions, the system Hamiltonian $H_{\text {sys }}$ was omitted in Eq. (5).

Using the gradient-ascent method for updating the control amplitudes $\theta_{m}$ increases the performance function but leaves the pulse ordering defined via the indices $k_{m}$ unchanged. Thus, for the optimum pulse order to be included in the configuration space, the number of pulses $M$ needs to be much larger than the expected minimum number of pulses finally realizing the target operation $U_{\text {target }}$. Therefore, the search algorithm has to be complemented by a penalty function like $\Phi_{p}=\sum_{m=1}^{M}\left|\theta_{m}\right|^{\gamma}$, $0<\gamma<1$, that tries to eliminate short pulses that do not contribute much to increasing the performance function $\Phi$. The functional form of $\Phi_{p}$ assures that a change in the length $\theta_{m}$ of the $m$ 'th pulse by $d \theta_{m}$ penalizes already short pulses much more than longer ones since $d \Phi_{p}=\operatorname{sign}\left(\theta_{m}\right) \gamma\left|\theta_{m}\right|^{\gamma-1} d \theta_{m}$. In the optimization routine used for finding the pulse decompositions presented in section IV] the exponent $\gamma$ ranged from 0.5 to 0.8 . Now, the performance function $\Phi$ is replaced by $\hat{\Phi}=\Phi-\alpha \Phi_{p}$ where $\alpha$ is a suitably chosen weight.

For updating the pulse lengths $\theta_{m}$, we do not calculate the gradient of $\hat{\Phi}$ and move in the direction of steepest ascent but perform consecutive one-dimensional maximizations of $\theta_{m}$ instead. This has the advantage that the step size $\epsilon$ of Eq. (4) can be individually adjusted for the different directions by considering also the curvatures $\partial^{2} \hat{\Phi} / \partial \theta_{m}^{2}$. For a negative curvature, the pulse length $\theta_{m}$ is updated by going to the maximum of the parabola approximating the $\Phi$ whereas for a positive curvature a fixed step size is used for updating $\theta_{m}$.

To avoid becoming trapped in a local maximum of the performance function, we combine the uphill search algorithm with elements of simulated annealing. Instead of choosing the pulse length $\theta_{m}^{*}$ corresponding to the maximum of the parabolic approximation to $\hat{\Phi}$, the algorithm samples the region around the maximum by randomly choosing a pulse length $\theta_{m}=\theta_{m}^{*}+\Delta \theta$ where $\Delta \theta$ is randomly drawn from a normal distribution with probability density $\propto \exp \left(\left(\partial^{2} \hat{\Phi} / \partial \theta_{m}^{2}\right)(\Delta \theta)^{2} / T_{\text {eff }}\right)$ where the effective temperature $T_{\text {eff }}$ determines the spread of the distribution around $\theta_{m}^{*}$. In the course of the optimization, $T_{\mathrm{eff}}$ is lowered to zero. In addition, the algorithm tries to introduce new pulses into the sequence from time to time to achieve a variation of the pulse order. Unless otherwise mentioned the program is started from a random sequence of pulses of sufficient length.

The computational overhead for performing an update of the pulse amplitudes is the same for a method following the gradient and for the $M$ consecutive onedimensional optimizations of the pulse lengths. Choosing the one-dimensional optimizations allows us to calculate the curvatures $\partial^{2} \hat{\Phi} / \partial \theta_{m}^{2}$ at no additional cost as well as to include the annealing technique. It would be preferable to use all the information encoded in the elements $\partial^{2} \hat{\Phi} / \partial \theta_{m} \partial \theta_{n}$ of the Hesse matrix, however, we judged the computational cost amounting to $\mathcal{O}\left(M^{2}\right)$ matrix multiplications to be prohibitively high.

\section{EXAMPLES}

The optimization routine was used to search for decompositions of unitary transformations of interest in systems of three to five qubits. In the following subsections, examples will be given for pulse sequences found by the program. Interestingly, it turns out that in most cases the sequences consist of pulses having pulse lengths that are simple fractions of $\pi$ even though the optimization routine was allowed to vary the pulse lengths continu- 
ously. Moreover, the pulse sequences listed below realize the desired target operation not only approximately but exactly.

Depending on the initial pulse sequence and the values of the parameters controlling the optimization process, the optimization algorithm can converge to different solutions realizing a target operation $U_{\text {target }}$. This demonstrates that we cannot be sure that the pulse sequences found by the program necessarily represent the optimum solution. However, as we are interested in discovering sequences of practical interest to be used in experiments, this is hardly a drawback.

In the following, we simplify our notation by using the short-hand notation $U_{z}^{(k)}(\theta) \leftrightarrow[\theta]_{z}^{k}, U_{X}(\theta) \leftrightarrow[\theta]_{X}$, $U_{M S}^{X}(\theta) \leftrightarrow[\theta]_{X X}$ to achieve a convenient and compact representation of the pulse sequences. Pulses are separated by hyphens and temporally ordered from left to right. Whenever a sequence is given, the number of qubits it is operating on is mentioned in the text.

\section{A. CNOT gates on three qubits}

In a system of three qubits, a CNOT gate between two qubits is realized by the sequence

$$
\begin{aligned}
& {\left[\frac{\pi}{2}\right]_{X}-\left[\frac{\pi}{2}\right]_{z}^{1}-\left[\frac{\pi}{4}\right]_{X X}-\left[\frac{\pi}{4}\right]_{X}-[\pi]_{z}^{3}-\left[\frac{\pi}{4}\right]_{X}-} \\
& {\left[\frac{\pi}{4}\right]_{X X}-\left[\frac{\pi}{2}\right]_{z}^{1}-\left[\frac{\pi}{2}\right]_{X}-[\pi]_{z}^{3},}
\end{aligned}
$$

where the target qubit 2 is controlled by qubit 1 , corresponding to the operation $U=\left(\mathcal{I}+\sigma_{z}^{(1)}+\sigma_{x}^{(2)}-\right.$ $\left.\sigma_{z}^{(1)} \sigma_{x}^{(2)}\right) / 2$.

A unitary transformation consisting of two CNOT operations with qubit 1 controlling the other two qubits is described by the unitary operation $U=\left(\mathcal{I}+\sigma_{z}^{(1)}+\right.$ $\left.\sigma_{x}^{(2)} \sigma_{x}^{(3)}-\sigma_{z}^{(1)} \sigma_{x}^{(2)} \sigma_{x}^{(3)}\right) / 2$. One way of decomposing it into elementary operations is given by the sequence

$$
\begin{aligned}
& {\left[\frac{\pi}{2}\right]_{X}-\left[-\frac{\pi}{2}\right]_{z}^{1}-\left[\frac{\pi}{4}\right]_{X X}-\left[-\frac{\pi}{4}\right]_{X}-[\pi]_{z}^{1}-\left[-\frac{\pi}{4}\right]_{X}-} \\
& {\left[-\frac{\pi}{4}\right]_{X X}-\left[\frac{\pi}{2}\right]_{z}^{1}-\left[\frac{\pi}{2}\right]_{X} .}
\end{aligned}
$$

As an example of a pulse sequence found by the search algorithm that is not composed of pulses having pulse angles which are simple rational fractions of $\pi$, we present another decomposition of the three-qubit operation $U$ given by

$$
\left[\beta_{2}\right]_{X X}-\left[\alpha_{2}\right]_{z}^{1}-\left[\beta_{2}\right]_{X X}-\left[\alpha_{1}\right]_{z}^{1}-\left[\beta_{1}\right]_{X X}-\left[\alpha_{1}\right]_{z}^{1} .
$$

For $\alpha_{1} \approx 0.7121 \pi, \alpha_{2} \approx-0.4241 \pi, \beta_{1} \approx-0.2121 \pi$ and $\beta_{2} \approx 0.3560 \pi$, it also realizes up to an unimportant global phase two CNOT gates on three qubits with qubit 1 controlling the other two. The program having provided the pulse angles, we found in a second step that the angles satisfy the algebraic relations $\alpha_{1}=2 \beta_{2}, \alpha_{2}=\pi-4 \beta_{2}$, $\beta_{1}=\pi / 2-2 \beta_{2}$ with $\beta_{2}=\frac{3}{8} \pi-\frac{1}{4} \arcsin (\sqrt{5}-2)$.

\section{B. CNOT gates on more than three qubits}

In general, a sequence realizing a two-qubit gate in a system with $N=3$ qubits will not correctly function in the case $N>3$ as it risks to entangle the spectator qubits with each other. On the other hand, a sequence realizing a two-qubit gate for $N=4$ which does not contain any phase shift gates on the spectator qubits is also applicable to $N>4$ because of the symmetry of the interactions between the different spectator qubits. A pulse sequence realizing a CNOT gate for $N \geq 4$ is given by

$$
\begin{aligned}
& {\left[\frac{\pi}{2}\right]_{X}-\left[-\frac{\pi}{2}\right]_{z}^{1}-\left[-\frac{\pi}{8}\right]_{X X}-[\pi]_{z}^{2}-\left[\frac{\pi}{8}\right]_{X X}-} \\
& {\left[\frac{\pi}{4}\right]_{X}-[\pi]_{z}^{1}-\left[-\frac{\pi}{8}\right]_{X X}-[\pi]_{z}^{2}-\left[\frac{\pi}{8}\right]_{X X}-} \\
& {\left[-\frac{\pi}{4}\right]_{X}-\left[-\frac{\pi}{2}\right]_{z}^{1}-\left[-\frac{\pi}{2}\right]_{X}}
\end{aligned}
$$

where again the qubit 1 controls the target qubit 2 .

\section{Further multi-qubit operations}

Apart from CNOT gate operations, we also searched for a decomposition of a quantum Toffoli gate operation. The sequence [22]

$$
\begin{aligned}
& {\left[\frac{\pi}{2}\right]_{Y}-\left[\frac{\pi}{4}\right]_{z}^{3}-\left[\frac{\pi}{2}\right]_{X X}-\left[-\frac{\pi}{2}\right]_{X}-\left[-\frac{\pi}{2}\right]_{z}^{3}-\left[-\frac{\pi}{4}\right]_{X}-} \\
& {\left[\frac{\pi}{4}\right]_{X X}-\left[\frac{\pi}{2}\right]_{z}^{3}-\left[\frac{\pi}{2}\right]_{X X}-\left[\frac{\pi}{2}\right]_{X}-\left[-\frac{\pi}{2}\right]_{Y}}
\end{aligned}
$$

is applicable to a system of three qubits and flips the state of the third qubit depending on the state of qubits 1 and 2 , thus realizing the operation $U=\left(3 \mathcal{I}+\sigma_{x}^{(3)}+\right.$ $\left.\left(\sigma_{z}^{(1)}+\sigma_{z}^{(2)}-\sigma_{z}^{(1)} \sigma_{z}^{(2)}\right)\left(\mathcal{I}-\sigma_{x}^{(3)}\right)\right) / 4$.

In a system with an even number $N=2 M$ of qubits, a mapping between the Bell basis and the product state basis for each pair of qubits $(2 m-1,2 m), m=1, \ldots M$, is of interest as it could be used for measuring the multipartite concurrence of an $M$-qubit quantum state available in two copies 23] and for implementing entanglement purification protocols. For the case of four qubits, the sequence

$$
\left[\frac{\pi}{4}\right]_{X X}-[\pi]_{z}^{1}-[\pi]_{z}^{2}-\left[\frac{\pi}{4}\right]_{X X}-[\pi]_{z}^{1}-[\pi]_{z}^{2}
$$

realizes the desired mapping described by $U_{(1-2,3-4)}=$ $\exp \left(-i \frac{\pi}{4}\left(\sigma_{x}^{(1)} \sigma_{x}^{(2)}+\sigma_{x}^{(3)} \sigma_{x}^{(4)}\right)\right)$.

The approach can be extended to higher numbers of qubits. Executing the sequence

$$
\begin{aligned}
& {\left[\frac{\pi}{8}\right]_{X X}-[\pi]_{z}^{1}-[\pi]_{z}^{2}-[\pi]_{z}^{3}-[\pi]_{z}^{4}-} \\
& {\left[\frac{\pi}{8}\right]_{X X}-[\pi]_{z}^{1}-[\pi]_{z}^{2}-[\pi]_{z}^{5}-[\pi]_{z}^{6}}
\end{aligned}
$$

twice, realizes the unitary transformation $U_{(1-2,3-4, \ldots)}=$ $\exp \left(-i \frac{\pi}{4} \sum_{m=1}^{M} \sigma_{x}^{(2 m-1)} \sigma_{x}^{(2 m)}\right)$ for the case $N=6,8$. 


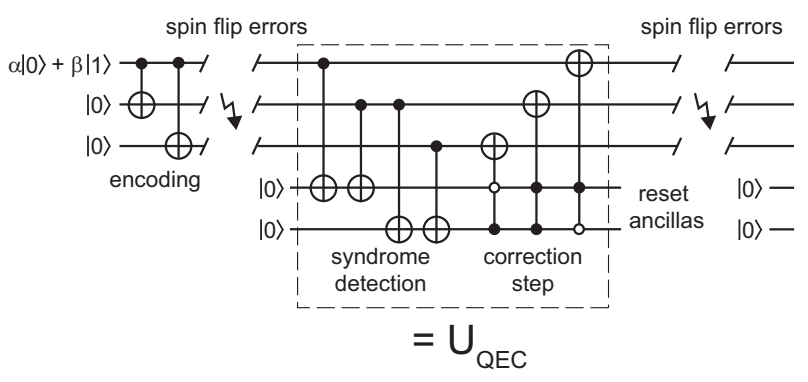

FIG. 1: Repetitive error correction of spin flips using three qubit for encoding the logical qubit and two ancilla qubits for error syndrome detection and coherent correction of errors. The optimal control algorithm looked for a decomposition of the unitary operation enclosed in the dashed box comprising syndrome detection and error correction.

Moreover, this approach could also be used to create linear cluster states and to realize the Hamiltonian of a 1-D Ising model by concatenating the unitaries $U_{(1-2,3-4, \ldots)}$ and $U_{(2-3,4-5, \ldots)}$.

\section{Quantum error correction}

In experimental quantum information processing, first steps have been taken to demonstrate quantum error correction [24, 25] using three-qubit codes. A major step forward would be the realization of repetitive error correction where the quantum information remains encoded in a logical qubit all the time. In the example shown in Fig. 1. a qubit state $\alpha|0\rangle+\beta|1\rangle$ is encoded in a logical qubit consisting of three qubits as $\left|\psi_{L}\right\rangle=\alpha|000\rangle+\beta|111\rangle$. The circuit detects single bit flip errors $\sigma_{x}^{(m)}, m=1,2,3$, by means of two additional ancilla qubits for syndrome detection and corrects the errors coherently by application of quantum Toffoli gates in order to restore the state
$\left|\psi_{L}\right\rangle$.

Using gate decompositions similar to Eq. (6) for constructing the unitary transformation $U_{\mathrm{QEC}}$ that detects and corrects errors from CNOT and Toffoli gates would result in a pulse sequence with more than 100 pulses that does not seem to be realizable with current technology. Therefore, the only practical approach seems to be to search for a gate decomposition of the complete operation $U_{\mathrm{QEC}}$. However, as the time needed for finding gate decompositions scales exponentially with the number of qubits, the task of decomposing the five-qubit operation $U_{\mathrm{QEC}}$ might seem quite challenging at a first glance. But actually, there is a whole class of operations $\tilde{U}_{\mathrm{QEC}}$ equivalent to $U_{\mathrm{QEC}}$ accomplishing the error correction protocol. To see this, note that we only require $\tilde{U}_{\mathrm{QEC}}$ to perform the mapping $U^{(m)}\left|\psi_{L}\right\rangle|00\rangle \rightarrow\left|\psi_{L}\right\rangle\left|\psi_{A}^{(m)}\right\rangle$ where $U^{(m)} \in\left\{\mathcal{I}, \sigma_{x}^{(1)}, \sigma_{x}^{(2)}, \sigma_{x}^{(3)}\right\}$ and $\left|\psi_{A}^{(m)}\right\rangle$ are arbitrary orthonormal vectors describing the ancilla state at the end of the correction step. Therefore, any valid transformation $\tilde{U}_{\mathrm{QEC}}$ can be put into the form

$$
\begin{aligned}
\tilde{U}_{\mathrm{QEC}} P_{00}^{A}= & \sum_{m}\left(|000\rangle\left\langle 000\left|U_{m} \otimes\right| \psi_{A, 0}^{m}\right\rangle\langle 00|\right. \\
& \left.+|111\rangle\left\langle 111\left|U_{m} \otimes\right| \psi_{A, 1}^{m}\right\rangle\langle 00|\right)
\end{aligned}
$$

where the ancilla states needs to satisfy the constraint $\psi_{A, 0}^{m}=\psi_{A, 1}^{m}$. Here, $P_{00}^{A}=\mathcal{I} \otimes|00\rangle\langle 00|$ is a projector onto the initial ancilla state. The condition $\left\langle\psi_{A, 0} \mid \psi_{A, 1}\right\rangle=1$ expressing the constraint $\psi_{A, 0}^{m}=\psi_{A, 1}^{m}$ assures that no information about the state of the logical qubit can be obtained from detecting the ancilla state. To search for a gate decomposition, the performance function can now be modified by replacing the trace $\operatorname{Tr}\left(U^{\dagger} U_{\mathrm{QEC}}\right)$ by $\Phi=$ $\operatorname{Re}\left(\sum_{m}\left\langle\psi_{A, 0}^{(m)} \mid \psi_{A, 1}^{(m)}\right\rangle\right)$.

Using this approach, the search program found the following pulse decomposition $\tilde{U}_{\mathrm{QEC}}$ for realizing an operation equivalent to $U_{\mathrm{QEC}}$ :

$$
\begin{aligned}
& {\left[-\frac{\pi}{2}\right]_{X}-\left[\frac{\pi}{2}\right]_{z}^{5}-\left[\frac{\pi}{2}\right]_{z}^{4}-[\pi]_{z}^{3}-\left[-\frac{\pi}{8}\right]_{Y Y}-[\pi]_{z}^{2}-\left[-\frac{\pi}{8}\right]_{Y Y}-[\pi]_{z}^{5}-[\pi]_{z}^{3}-\left[\frac{\pi}{8}\right]_{Y Y}-[\pi]_{z}^{2}-\left[-\frac{3 \pi}{8}\right]_{Y Y}-\left[\frac{\pi}{2}\right]_{X}-\left[\frac{\pi}{2}\right]_{z}^{3}-} \\
& {\left[\frac{\pi}{2}\right]_{z}^{1}-\left[-\frac{\pi}{8}\right]_{X X}-[\pi]_{z}^{5}-[\pi]_{z}^{4}-\left[\frac{\pi}{8}\right]_{X X}-[\pi]_{z}^{1}-\left[\frac{\pi}{2}\right]_{z}^{4}-[\pi]_{z}^{5}-\left[\frac{\pi}{8}\right]_{X X}-[\pi]_{z}^{2}-\left[-\frac{3 \pi}{8}\right]_{X}-[\pi]_{z}^{4}-\left[-\frac{\pi}{8}\right]_{X X}-[\pi]_{z}^{5}-} \\
& {\left[\frac{\pi}{8}\right]_{X X}-\left[\frac{\pi}{8}\right]_{X}-[\pi]_{z}^{2}-\left[\frac{\pi}{8}\right]_{X X}-\left[-\frac{\pi}{2}\right]_{z}^{4}-\left[-\frac{\pi}{4}\right]_{X X}}
\end{aligned}
$$

Here, the uphill search was extremely slow when starting the search algorithm from a random sequence of pulse. However, starting the algorithm from a sequence constructed from gate decompositions of the CNOT and Toffoli gates and initially driving it away from this undesired solution by increasing the effective temperature $T_{\text {eff }}$ proved to be an effective strategy for finding an improved solution.

\section{SUMMARY AND OUTLOOK}

In conclusion, we have developed an algorithm based on optimal control techniques for finding decompositions 
of unitary transformations into finite sequences of pulses that correspond to the application of Hamiltonians drawn from a given set. For a set of Hamiltonians that are of interest for ion trap quantum computing, the algorithm provides gate decompositions that would be difficult to find otherwise.

Looking for gate decompositions involving parallel twoqubit interactions on a small number of ions provided in many cases (as for example for the quantum Toffoli gate) a pulse sequence much shorter than what would have been possible by using sequential two-qubit interactions and single qubit gates. In a few other cases, however, this strategy did not pay off (as we noted when looking for a decomposition of the quantum Fredkin gate).

While our investigation was limited to a particular basis set of Hamiltonians $\mathcal{S}$, the program could be adapted to search for gate decompositions using other sets that might be more relevant for realizations of quantum computing in other physical systems where, for example, the natural entangling interaction is given by a $\sqrt{i \text { SWAP }}$ gate [26] or an exchange interaction [27]. For example, in the context of ion trap quantum computing, another interesting question is whether the algorithm could be modified to make it applicable to the case of quantum gates realized by a tightly focussed laser interacting with a single ion at a time. In this case, the Hilbert space would be comprised not only of the qubit states but also of the harmonic oscillator the qubits are coupling to, a configuration not only found in ion trap quantum computing but also in cavity-QED setups where an electromagnetic field mode is interacting with cold atoms or superconducting flux qubits.

\section{Acknowledgments}

We gratefully acknowledge the support of the European network SCALA, the Institut für Quanteninformation $\mathrm{GmbH}$ and IARPA. C. F. R. would like to thank T. Schulte-Herbrüggen and S. Glaser for useful discussions and C. Kruszynska for help with symbolic calculations.
[1] D. Deutsch, Proc. R. Soc. Lond. A 400, 97 (1985).

[2] M. A. Nielsen and I. L. Chuang, Quantum Computation and Quantum Information (Cambridge Univ. Press, Cambridge, 2000).

[3] A. Barenco, C. H. Bennett, R. Cleve, D. P. DiVincenzo, N. Margolus, P. Shor, T. Sleator, J. A. Smolin, and H. Weinfurter, Phys. Rev. A 52, 3457 (1995).

[4] S. Lloyd, Phys. Rev. Lett. 75, 346 (1995).

[5] R. Blatt and D. Wineland, Nature 453, 1008 (2008).

[6] H. C. Nägerl, D. Leibfried, H. Rohde, G. Thalhammer, J. Eschner, F. Schmidt-Kaler, and R. Blatt, Phys. Rev. A 60, 145 (1999).

[7] F. Schmidt-Kaler, H. Häffner, S. Gulde, M. Riebe, G. P. T. Lancaster, T. Deuschle, C. Becher, W. Hänsel, J. Eschner, C. F. Roos, et al., Appl. Phys. B 77, 789 (2003).

[8] D. Leibfried, B. DeMarco, V. Meyer, D. Lucas, M. Barrett, J. Britton, W. M. Itano, B. Jelenković, C. Langer, T. Rosenband, et al., Nature 422, 412 (2003).

[9] C. A. Sackett, D. Kielpinski, B. E. King, C. Langer, V. Meyer, C. J. Myatt, M. Rowe, Q. A. Turchette, W. M. Itano, D. J. Wineland, et al., Nature 404, 256 (2000).

[10] J. Benhelm, G. Kirchmair, C. F. Roos, and R. Blatt, Nat. Phys. 4, 463 (2008).

[11] Assuming that a single-qubit gate is performed with a laser beam with intensity $I$ and a Gaussian spatial beam profile with waist $w$, it is advantageous to work with an interaction whose strengths falls off as $\theta(r) \propto$ $\exp \left(-2 r^{2} / w^{2}\right)$ instead of $\theta(r) \propto \sqrt{I} \propto \exp \left(-r^{2} / w^{2}\right)$.

[12] M. A. Rowe, A. Ben-Kish, B. DeMarco, D. Leibfried, V. Meyer, J. Beall, J. Britton, J. Hughes, W. M. Itano, B. Jelenković, et al., Quant. Inf. Comp. 2, 257 (2002).

[13] N. Khaneja, T. Reiss, C. Kehlet, T. Schulte-Herbrüggen, and S. J. Glaser, J. Magn. Reson. 172, 296 (2005).

[14] C. M. Tesch and R. de Vivie-Riedle, Phys. Rev. Lett. 89,
157901 (2002).

[15] J. P. Palao and R. Kosloff, Phys. Rev. A 68, 062308 (2003).

[16] L. M. K. Vandersypen and I. L. Chuang, Rev. Mod. Phys. 76, 1037 (2004).

[17] M. Grace, C. Brif, H. Rabitz, I. Walmsley, R. Kosut, and D. Lidar, J. Phys. B 40, S103 (2007).

[18] S. Montangero, T. Calarco, and R. Fazio, Phys. Rev. Lett. 99, 170501 (2007).

[19] G. D. Chiara, T. Calarco, M. Anderlini, S. Montangero, P. J. Lee, B. L. Brown, W. D. Phillips, and J. V. Porto, Phys. Rev. A 77, 052333 (2008).

[20] N. Timoney, V. Elman, S. Glaser, C. Weiss, M. Johanning, W. Neuhauser, and C. Wunderlich, Phys. Rev. A 77, 052334 (2008).

[21] If the sum in eq. (2) consists of only a single term, then eq. (3) becomes exact.

[22] As the Hamiltonians in set $\mathcal{S}$ are sufficient for generating arbitrary unitary operations, we did not include the Hamiltonians $S_{Y}, S_{Y}^{2}$. However, as these Hamiltonians are experimentally as easily generated as $S_{X}, S_{X}^{2}$ by shifting the optical phase of the control laser field by $\pi / 2$ with the help of an acousto-optical modulator, we use them in some of the pulse sequences for constructing the desired target operation.

[23] L. Aolita and F. Mintert, Phys. Rev. Lett. 97, 050501 (2006).

[24] D. G. Cory, M. D. Price, W. Maas, E. Knill, R. Laflamme, W. H. Zurek, T. F. Havel, and S. S. Somaroo, Phys. Rev. Lett. 81, 2152 (1998).

[25] J. Chiaverini, D. Leibfried, T. Schaetz, M. D. Barrett, R. B. Blakestad, J. Britton, W. M. Itano, J. D. Jost, E. Knill, C. Langer, et al., Nature 432, 602 (2004).

[26] M. Steffen, M. Ansmann, R. C. Bialczak, N. Katz, E. Lucero, R. McDermott, M. Neeley, E. M. Weig, A. N. 
Cleland, and J. M. Martinis, Science 313, 1423 (2006).

[27] D. P. DiVincenzo, D. Bacon, J. Kempe, G. Burkard, and
K. B. Whaley, Nature 408, 339 (2000). 